\title{
WHEELSET/RAIL GEOMETRIC CHARACTERISTICS ASSESSMENT WITH REGARD TO WHEELSET ROLLING
}

The geometric relation between a wheelset and a rail is assessed with the help of geometric characteristics. Geometric characteristics are: equivalent conicity function, delta $r$ function placement of contact points of a wheelset and a rail, tangent gamma function and effective conicity.

It turned out that these characteristics are at present the most important not only for the judgment of ride characteristics of a vehicle on the rail but also for the wearing of wheel treads and rail heads, e. i., for the assessment of the track and vehicles in order to find out the current state and for the assessment of changes of the wheels and rails profile shapes in order to improve the current state too. The process of geometric characteristic assessment of a wheelset and rail with regard to wheelset rolling is analysed in the article.

\section{Introduction}

Wheelset rolling is determined by the geometric position of a wheelset with regard to a track.

The profiles geometry and the lateral movement of a wheelset have an influence on the contact points position and on the wheelset rolling, as well as on other geometric characteristics for which the contact points represent input data.

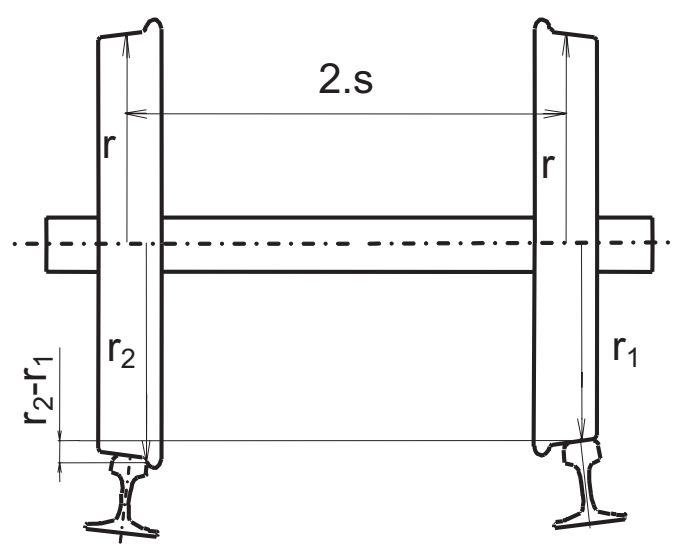

Fig. 1 Difference of wheels rolling immediate radii of one wheelset at its lateral movement.

For further analyses we arise from the assumption that the lateral movement of the profiles, not the point of gravity of the wheelset movement, is important.

It means, that we understand under the term of the lateral translation movement $y$ the parallel translation of wheels profiles coordinate system, not the lateral translation of the wheelset coordinate system itself. The point of gravity of the wheelset can move in a lateral direction by values different from the lateral movement on the high level of contact points. This happens with regard to the geometry of points in contact. In extreme cases the movement of the point of gravity can move in an opposite direction as "the profiles".

Wheelset point of gravity in the y-direction

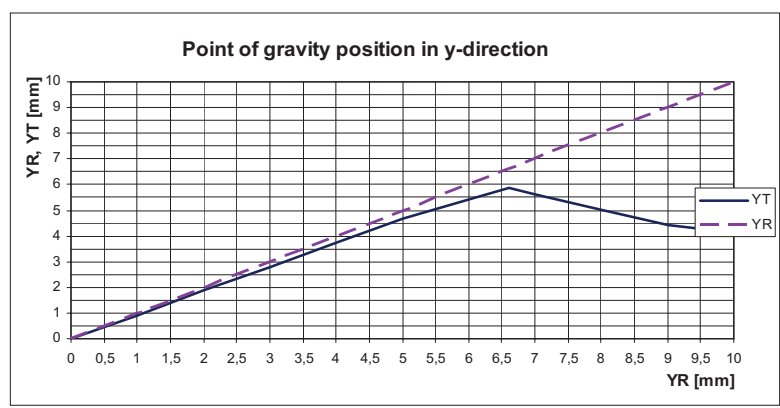

Fig. 2 Wheelset point of gravity position YT with regard to the profile movement in the $y$-direction, for the S1002/UIC60/1:40 profiles.

The wheel profiles move on the rail profiles in a lateral direction $(Y R)$. Contact points of the profile couples wheel/rail are found. The final rolling is determined on the base of a contact point on the wheel tread and of a wheel radius. In Fig. 2 is $Y R(Y R)$ dependence curve and $Y T(Y R)$ dependence curve difference.

\section{The geometric characteristics analysis process}

The wheelset profiles transformation via turning around contact points by the $\varphi$ angle.

The wheelset rolling is calculated in the following way:

\footnotetext{
* Juraj Gerlici, Tomáš Lack

Faculty of Mechanical Engineering, University of Žilina, Univerzitná 8215/1, SK-010 26 Žilina, Slovakia,

E-mail: juraj.gerlici@fstroj.utc.sk, tomas.lack@fstroj.utc.sk
} 
A wheelset is placed into a track at a certain $y_{R}$ lateral movement. system.

The right and left profiles are in the wheelset coordinate

Contact points of the wheelset and the track at the rolling angle $\varphi=0$ are found.

The minimum value of the function of difference $d$ determines the contact point position between the wheel and the rail.

$$
d\left(y, y_{R}\right)=z_{S}(y)-z_{R}\left(y-y_{R}\right)
$$

The $y$-coordinate of a contact point on the rail at the wheelset profile movement is the $y$-coordinate of the function of difference $d$ when the function of difference $d$ reaches the extreme (minimum).

$$
\left[Y_{S}\left(y_{R}\right)=y\right]_{d=}^{\min (d)} \underset{y \in(\text { WheelnRail })}{ }
$$

The minimum value of the function of difference $d$ at the wheel profile movement $y_{R}$ equals the value of the function of difference $d$ at the $y$-direction coordinate which equals at the same time the $y$-direction coordinate of the contact point on the rail profile.

$$
d_{\text {min }}\left(y_{R}\right)=\left[d\left(y, y_{R}\right)\right]_{y}=Y_{S}
$$

The whole wheelset preliminary tilts by the $\varphi$ angle on the base of the difference assessment of the size of immediate radii of wheels rolling:

$$
\varphi=\varphi_{0}+\operatorname{arctg}\left(\left(d_{1 \min }-d_{2 \min }\right) /(2 \cdot s)\right),
$$

$\varphi_{0}=0$ at the first cycle of computation,

$d_{1 \text { min }}=y$-direction coordinate of the right wheel contact point + right wheel radius,

$d_{2 \min }=y$-direction coordinate of the left wheel contact point + left wheel radius.

The wheelset rolling by $\varphi$ angle consists of summation of turning around the right wheel contact point by $\varphi / 2$ angle and turning around the left wheel contact point by $\varphi / 2$.

The right wheel profile turns by the $\varphi$ angle in the coordinate system with the beginning in the contact point of the left wheel and the rail (Fig. 4). The transformed profile is furthermore the $\varphi$ angle function. The contact point of the right wheel and the rail is found.

The left wheel profile turns by the $\varphi$ angle in the coordinate system with the beginning in the contact point of the right wheel and the rail (Fig. 5). The transformed profile is the $\varphi$ angle function. The contact point of the right wheel and the rail is found.

Parameters $d_{1 \min }$ a $d_{2 \min }$ are assessed again. If their difference is greater than the precision ratio $\varepsilon$ (when computing $10^{-6}$ ) the wheel profiles turn by the $\varphi$ angle according to the equation (3), while the value $\varphi_{0}$ is substituted by the $\varphi$ from the previous computation. The computation or the $\varphi$ angle is repeated until the difference of parameters $d_{1 \min }$ and $d_{2 \min }$ is lower than $\varepsilon$.

The inverse transformation of the wheelset contact points position into the coordinate system of the wheelset is realised via the turning by the $\varphi$ angle.

The geometric characteristics are evaluated.

The difference of geometric characteristics courses is determined with regard to the wheelset rolling from the geometric characteristics without rolling.

\section{Contact point searching}

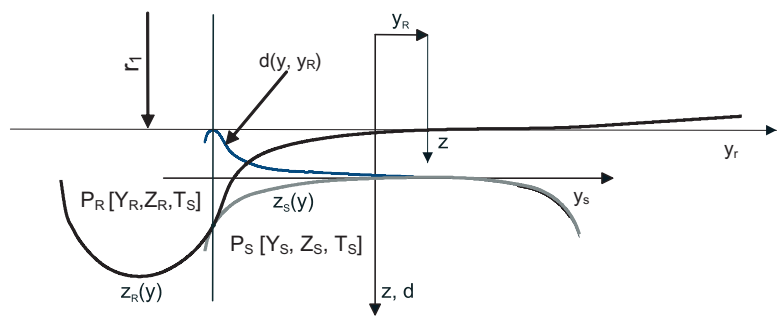

Fig. 3 Contact point of the right wheel and rail

$d\left(y, y_{R}\right) \quad$ function of difference of the z-direction coordinate of profiles,

$P_{S}\left[Y_{S}, Z_{S}, T_{S}\right]$ contact point of a rail,

$P_{R}\left[Y_{R}, Z_{R}, T_{R}\right]$ contact point of a wheel,

$Z\left(P_{R}\right) \quad$ Z- direction coordinate of a contact point of a wheel,

$z_{R} \quad$ profile of a wheel,

$z_{S} \quad$ profile of a rail,

$y_{R} \quad$ movement of a wheel profile.

\section{Profiles transformations}

\subsection{Profile transformation by turning around the left wheel contact point}

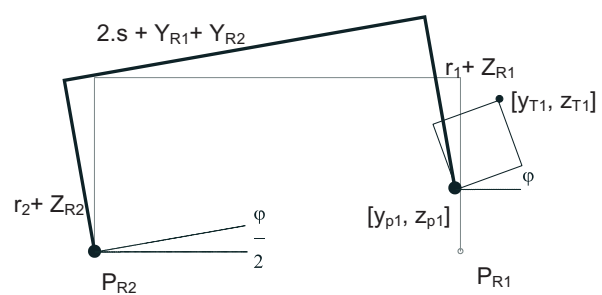

Fig. 4 Left contact point - wheels profiles transformations.

$$
\left\{\begin{array}{l}
y_{p 1} \\
z_{p 1}
\end{array}\right\}=\left[\begin{array}{rr}
\cos \left(\frac{\varphi}{2}\right)-1 & \sin \left(\frac{\varphi}{2}\right) \\
\sin \left(\frac{\varphi}{2}\right) & 1-\cos \left(\frac{\varphi}{2}\right)
\end{array}\right] .
$$


$\cdot\left\{\begin{array}{c}2 \cdot s+Y_{R 1}+Y_{R 2} \\ r_{2}+Z_{R 2}-\left(r_{1}+Z_{R 1}\right)\end{array}\right\}$

$$
\left\{\begin{array}{c}
y_{T 1} \\
z_{T 1} \\
1
\end{array}\right\}=\left[\begin{array}{ccc}
\cos (\varphi) & -\sin (\varphi) & y_{p 1}+Y_{R 1} \\
\sin (\varphi) & \cos (\varphi) & z_{p 1}+Z_{R 1} \\
0 & 0 & 1
\end{array}\right] \cdot\left\{\begin{array}{c}
y_{1}+Y_{R 1} \\
z_{1}-Z_{R 1} \\
1
\end{array}\right\}
$$

$\begin{array}{ll}s & \text { half taping line distance, } \\ r_{1} & \text { right wheel radius, } \\ r_{2} & \text { left wheel radius, } \\ z_{R 1}(y) & \text { right wheel profile function, } \\ z_{R 2}(y) & \text { left wheel profile function, } \\ \varphi & \text { turning angle, } \\ P_{R 1}\left[Y_{R 1}, Z_{R 1}, T_{R 1}\right] & \text { right wheel contact point, } \\ P_{R 2}\left[Y_{R 2}, Z_{R 2}, T_{R 2}\right] & \text { left wheel contact point, }\end{array}$

$z_{R 1 T}(y) \quad$ right wheel profile transformed function, $z_{R 2 T}(y) \quad$ left wheel profile transformed function.

\subsection{Profile transformation by turning around the right} wheel contact point

$$
\begin{aligned}
& \left\{\begin{array}{l}
y_{p 2} \\
z_{p 2}
\end{array}\right\}=\left[\begin{array}{cc}
\cos \left(\frac{\varphi}{2}\right)-1 & \sin \left(\frac{\varphi}{2}\right) \\
-\sin \left(\frac{\varphi}{2}\right) & \cos \left(\frac{\varphi}{2}\right)-1
\end{array}\right] . \\
& \cdot\left\{\begin{array}{c}
2 \cdot s+Y_{R 1}+Y_{R 2} \\
r_{2}+Z_{R 2}-\left(r_{1}+Z_{R 1}\right)
\end{array}\right\} \\
& \left\{\begin{array}{c}
y_{T 2} \\
z_{T 2} \\
1
\end{array}\right\}=\left[\begin{array}{ccc}
\cos (\varphi) & \sin (\varphi) & y_{p 2}+Y_{R 2} \\
-\sin (\varphi) & \cos (\varphi) & z_{p 2}+Z_{R 2} \\
0 & 0 & 1
\end{array}\right] \cdot\left\{\begin{array}{c}
y_{2}-Y_{R 2} \\
z_{2}-Z_{R 2} \\
1
\end{array}\right\}
\end{aligned}
$$

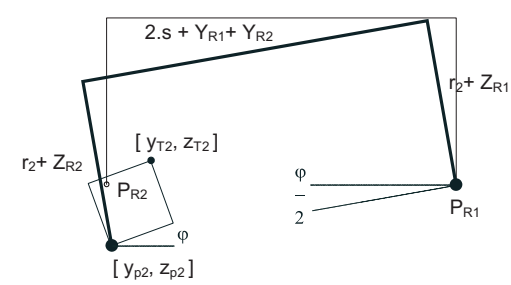

Fig. 5 Right contact point - wheels profiles transformations.

\section{Wheelset contact points inverse transformation} into the rail coordinate system via the turning by the $\varphi$ angle

In point 1 , contact points were found in the wheelset coordinate system. Now it is necessary to transform them into the rail coordinate system.

\subsection{Right wheel contact point inverse transformation}

$$
\begin{aligned}
& \left\{\begin{array}{l}
y_{p 1} \\
z_{p 1}
\end{array}\right\}=\left[\begin{array}{rr}
\cos \left(\frac{\varphi}{2}\right)-1 & \sin \left(\frac{\varphi}{2}\right) \\
\sin \left(\frac{\varphi}{2}\right) & 1-\cos \left(\frac{\varphi}{2}\right)
\end{array}\right] . \\
& \cdot\left\{\begin{array}{c}
2 \cdot s+Y_{R 1 O} \\
r_{2}+Z_{R 2 O}-\left(r_{1}+Z_{R 1 O}\right)
\end{array}\right\} \\
& \left\{\begin{array}{c}
y_{R 1} \\
z_{R 1} \\
1
\end{array}\right\}=\left[\begin{array}{ccc}
\cos (\varphi) & \sin (\varphi) & Y_{R 1 O} \\
-\sin (\varphi) & \cos (\varphi) & Z_{R 1 O} \\
0 & 0 & 1
\end{array}\right] . \\
& \cdot\left\{\begin{array}{c}
Y_{R 1}-y_{p 1}-Y_{R 1 O} \\
Z_{R 1}-z_{p 1}-Z_{R 1 O} \\
1
\end{array}\right\}
\end{aligned}
$$

\subsection{Left wheel contact point inverse transformation}

$$
\begin{aligned}
& \left\{\begin{array}{l}
y_{p 2} \\
z_{p 2}
\end{array}\right\}=\left[\begin{array}{cc}
\cos \left(\frac{\varphi}{2}\right)-1 & \sin \left(\frac{\varphi}{2}\right) \\
-\sin \left(\frac{\varphi}{2}\right) & \cos \left(\frac{\varphi}{2}\right)-1
\end{array}\right] . \\
& \cdot\left\{\begin{array}{c}
2 \cdot s+Y_{R 1 O}+Y_{R 2 O} \\
r_{2}+Z_{R 2 O}-\left(r_{1}+Z_{R 1 O}\right)
\end{array}\right\} \\
& \left\{\begin{array}{c}
y_{R 2} \\
z_{R 2} \\
1
\end{array}\right\}=\left[\begin{array}{ccc}
\cos (\varphi) & -\sin (\varphi) & Y_{R 2 O} \\
\sin (\varphi) & \cos (\varphi) & Z_{R 2 O} \\
0 & 0 & 1
\end{array}\right] \\
& \cdot\left\{\begin{array}{c}
Y_{R 2}-y_{p 2}-Y_{R 2 O} \\
Z_{R 2}-z_{p 2}-Z_{R 2 O} \\
1
\end{array}\right\}
\end{aligned}
$$

$P_{R 1 O}\left[Y_{R 1 O}, Z_{R 1 O}, T_{R 1 O}\right]$ - right wheel contact point from the previous iteration step,

$P_{R 2 O}\left[Y_{R 2 O}, Z_{R 2 O}, T_{R 2 O}\right]$ - left wheel contact point from the previous iteration step,

$P_{R 1}\left[Y_{R 1}, Z_{R 1}, T_{R 1}\right]$ - right wheel contact point turned by the $\varphi$ angle,

$P_{R 2}\left[Y_{R 2}, Z_{R 2}, T_{R 2}\right]-$ left wheel contact point turned by the $\varphi$ angle.

\section{Computation of the geometric characteristics at the wheelset rolling}

The separate points of the characteristics are determined step by step for $y=y_{R \min }$ to $y_{R \max }$ with the step $\Delta y_{R}$. The procedure suitable for computer processing is determined by the equations 13-30. 
1. $\varphi=0$

2. $d_{1 \min }=$ Contact point $\left(y, z_{S 1}, z_{R 1}, P_{S 1}, P_{R 1}\right)+r_{1}$

3. $d_{2 \min }=$ Contact $\operatorname{point}\left(y, z_{S 2}, z_{R 2}, P_{S 2}, P_{R 2}\right)+r_{2}$

4. $\varphi=\varphi+\operatorname{arctg}\left(\left(d_{1 \min }-d_{2 \min }\right) /(2 . s)\right)$

5. Saving of previous wheelset contact points

- $P_{R 1 O}=P_{R 1}$

- $P_{R 2 O}=P_{R 2}$

6. Transformation of wheelset profiles via their turning around the contact points by the $\varphi$ angle

$\left(s, r_{1}, r_{2}, z_{R 1}, z_{R 2}, P_{R 1}, P_{R 2}, \varphi, z_{R 1 T}, z_{R 2 T}\right)$

7. $d_{1 \mathrm{~min}}=$ Contact point $\left(y, z_{S 1}, z_{R 1 T}, P_{S 1}, P_{R 1}\right)+r_{1}$

8. $d_{2 \min }=$ Contact point $\left(y, z_{S 2}, z_{R 2 T}, P_{S 2}, P_{R 2}\right)+r_{2}$

9. If $A b s\left(d_{1 \text { min }}-d_{2 \min }\right)>1.10^{-6}$ go to point 4

10. Wheelset contact points inverse transformation

$\left(s, r_{1}, r_{2}, P_{R 1 O}, P_{R 2 O}, P_{R 1}, P_{R 2}, \varphi\right)$

- $P_{S 1}[y]=P_{S 1}$

- $P_{R 1}[y]=P_{R 1}$

- $P_{S 2}[y]=P_{S 2}$

- $P_{R 2}[y]=P_{R 2}$

11. $\Delta r[y]=r_{1}+Z_{R 1}-\left(r_{2}+Z_{R 2}\right)$

12. $T g_{1}[y]=T_{R 1}$

13. $\operatorname{Tg}_{2}[y]=-T_{R 2}$

14. $\operatorname{Tg}[y]=\operatorname{Tg}_{1}[y]+\operatorname{Tg}_{2}[y]$

15. $E k[y]=\frac{\Delta r[y]}{2 \cdot y}$ for $y<>0, E k[y]=0$ for $y=0$

16. $\varphi[y]=\varphi$

$z_{S 1}(y)$ right rail profile function,

$z_{S 2}(y)$ left rail profile function,

$P_{R 1} \quad$ right wheel contact point vector,

$P_{R 2} \quad$ left wheel contact point vector,

$y_{\text {Rmin }}$ minimum wheelset movement $(-10 \mathrm{~mm})$,

$y_{R \max }$ maximum wheelset movement $(10 \mathrm{~mm})$,

$\Delta r \quad \Delta \mathrm{r}$ function vector,

$\Delta y_{R} \quad$ wheelset movement step,

$P_{S 1} \quad$ right rail contact point vector,

$P_{S 2} \quad$ left rail contact point vector,

$\mathrm{Tg}_{1}$ tangent gamma function vector for the right wheel,

$\mathrm{Tg}_{2}$ tangent gamma function vector for the left wheel,

Ek effective conicity vector,

$\mathrm{Tg}$ tangent gamma function vector.

$\varphi \quad$ wheelset rolling vector,

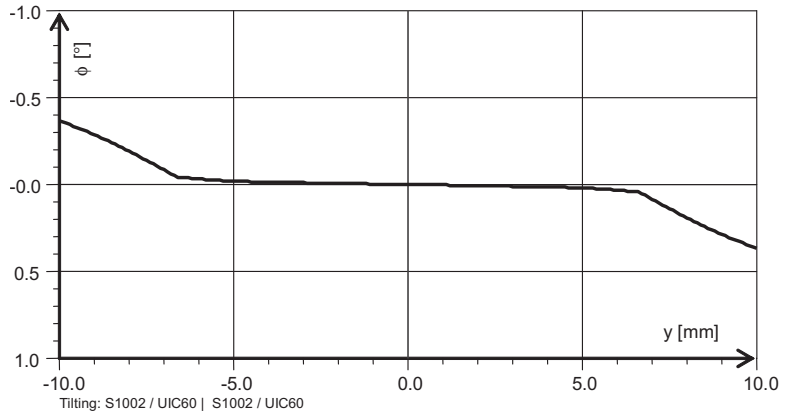

Fig. 6 Course of wheelset rolling angle.

\section{The influence of the wheelset rolling on the shape of geometric characteristics}

\subsection{Contact points}

The position of contact points between a wheelset and a rail influences the characteristics. The decisive factor which influences difference in the shape of characteristics is the difference of the contact point position in the case when the rolling is not taken into consideration and in the case when the wheelset rolling is taken into consideration.

In Fig. 7 the contact points distribution is shown (in this case standard combination of profiles S1002/UIC60/1:40/460) without regard to the rolling and in the Fig. 8 with regard to the rolling. In this case the difference is visible at the lateral wheelset movement greater than approximately $7 \mathrm{~mm}$.

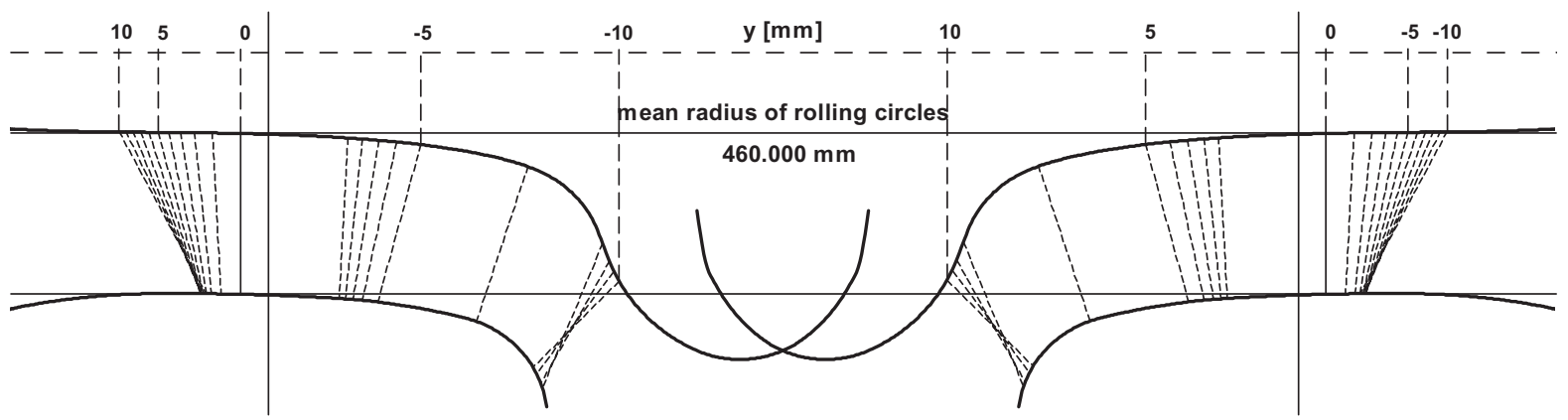

Fig. 7 Contact points distribution of non-rolled wheelset. 


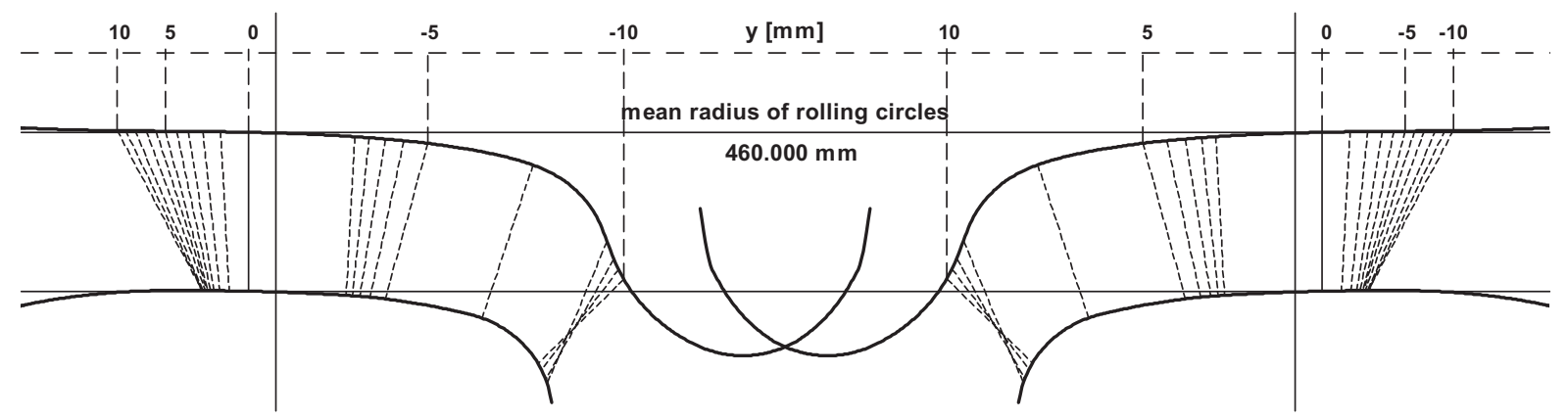

Fig. 8 Contact points distribution of rolled wheelset.

\section{2 delta $\mathbf{r}$ function, tangent gamma function}

The development of the $\Delta r$ function values when taking into consideration the wheelset rolling under the influence of different contact circles radii. tion with the rolling angle development and with the contact points distribution development. The differences are negligible for the amplitude range of a wheelset approximately $\langle-6,6\rangle$.

In this case, the other characteristics, effective and equivalent conicity, similar to the $\Delta r$ function and tangent gamma function

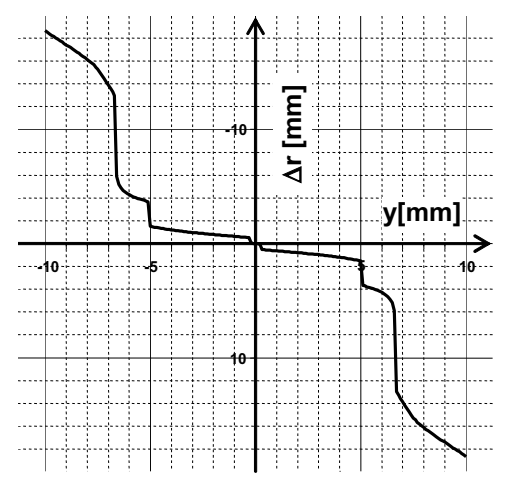

Fig. $9 \Delta$ r function.

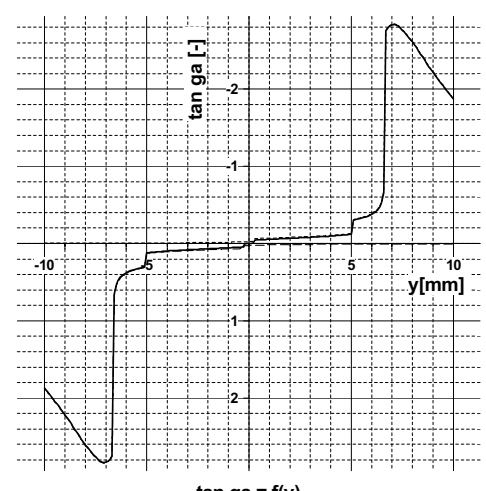

$\tan -g a=f(y)$

Fig.11 Tangent gamma (tan-ga) function.

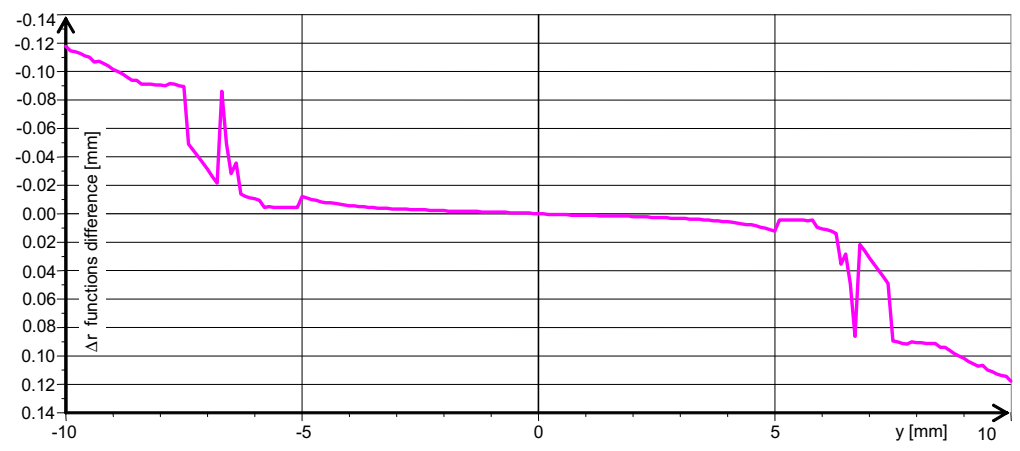

Fig.10 $\Delta r$ functions difference with regard to the wheelset rolling and without regard to wheelset rolling.

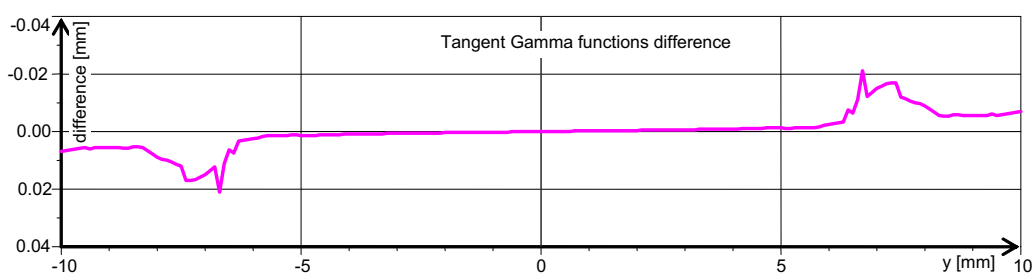

Fig. 12 Tangent gamma functions difference with regard to the wheelset rolling and without regard to wheelset rolling.
The differences in the $\Delta r$ function and tangent gamma function values are visible in the Fig. 10 and 12. They are in coordina- differ only slightly from characteristics computed without taking into consideration the wheelset rolling. 


\section{Conclusions}

The wheelset rolling at the lateral wheelset movement on the track expresses on the shape and size of geometric characteristics.

The significance of the change depends on the profiles shape of the wheel tread and on the rail head profile. When evaluating a standard couple of profiles: wheel S1002, rail UIC60, rail slope of 1:40 and the wheel radius $460 \mathrm{~mm}$, the influence of rolling expresses in the amplitude range $\langle-6,6\rangle$ to a minimum extension. The influence is negligible in the range $\langle-7,-6\rangle$ and $\langle 6,7\rangle$, when the wheelset is rolled to the value approximately $0.1^{\circ}$.

\section{References}

[1] GERLICI, J., LACK, T.: Contact of a railway wheelset and a rail (in Slovak). Scientific monograph, ISBN 80-8070-317-5, EDIS publishing house of University of Žilina, 2004.

[2] GERLICI, J., LACK, T.: Rail geometry analysis - from the point of view of wearing in the operation. Komunikácie - ved. listy Žilinskej univerzity, EDIS - publishing house of University of Žilina, 2003.

[3] GERLICI, J., LACK, T.: Railway wheel and rail geometry influence on ride properties of the vehicle (In Slovak). In: 16. International Conference PRORAIL 2003, Žilinská univerzita, EDIS - publishing house of University of Žilina, 2003.

[4] GERLICI, J., LACK, T., KADOROVÁ, M.: Calculation of the Equivalent Conicity Function with a Negative Slope. Komunikácie - ved. listy Žilinskej univerzity, EDIS - publishing house of University of Žilina, 2004.

[5] UIC Code 519: Method for determining the equivalent conicity. Draft of January 2003 Original version, 2003. 